\title{
Maternal Hypovitaminosis D as a Cause of neonatal seizure
}

\author{
Yoriko Nishizawa ${ }^{1}$, Sonam² ${ }^{2}$ Carolyn Mize $^{3}$, Tamara Vesel ${ }^{4}$ \\ ${ }^{1,2}$ Faculty of Postgraduate Medicine, Khesar Gyalpo University of Medical Sciences of Bhutan, Thimphu, Bhutan \\ ${ }^{3}$ Health Volunteers Overseas \\ ${ }^{4}$ Department of Medicine, Tufts Medical Center, Tufts Medical School, Boston, MA, USA
}

\begin{abstract}
Maternal hypovitaminosis D has many implications for both mother and newborn. The prevalence of vitamin D deficiency in Bhutan is not yet known. The case presented below represents a severe symptomatic presentation of vitamin D deficiency in a neonate due to maternal vitamin D deficiency. It highlights the need for raised suspicion of vitamin D deficiency as a cause of lateonset neonatal seizures, epidemiologic studies, and potential vitamin supplementation for pregnant women, as well as the need for vitamin D-only liquid supplements in pharmacies in Bhutan.
\end{abstract}

Keywords: Hypocalcemia; Maternal hypovitaminosis; Neonatal Seizure; Vitamin D deficiency.

\section{INTRODUCTION}

The neonatal period is the most vulnerable time throughout life for developing seizures. The prevalence of neonatal seizures is approximately $1.5 \%$ and overall incidence is approximately 3 per 1,000 live births ${ }^{1}$. The etiology of neonatal seizures is extensive (Table 1).

\section{Table 1. Etiology of neonatal seizure}

Hypoxic-ischemic encephalopathy (HIE)

Metabolic causes

Hypoglycemia

Hypocalcemia

Hypomagnesemia

Pyridoxine deficiency

Inborn errors of metabolism (IEM)

Infections

Meningitis

Meningoencephalitis secondary to intrauterine infections

(eg. TORCH group,syphilis)

Intracranial hemorrhage and infarction/stroke

Developmental defects

Cerebral dysgenesis

Neuronal migration disorders

Miscellancous

Polycythemia

Maternal narcotic withdrawal

Drug toxicity (eg. Theophylline, Doxapram)

Local anesthetic injection into scalp

Phacomatosis (eg. Tuberous Sclerosis,

Incontinentiapigmenti)

Corresponding author:

Yoriko Nishizawa

dryoriko@kgumsb.edu.bt
Hypocalcemia is a common metabolic problem in the neonatal period. The causes of neonatal hypocalcemia are classified by the timing of onset (Table 2). Late-onset hypocalcemia typically occurs at the end of the first week of life ${ }^{2,3}$. We present a case of a full-term infant who presented with hypocalcemic seizures due to severe maternal vitamin $\mathrm{D}$ deficiency.

Table 2. Etiology of hypocalcaemia in neonatal period

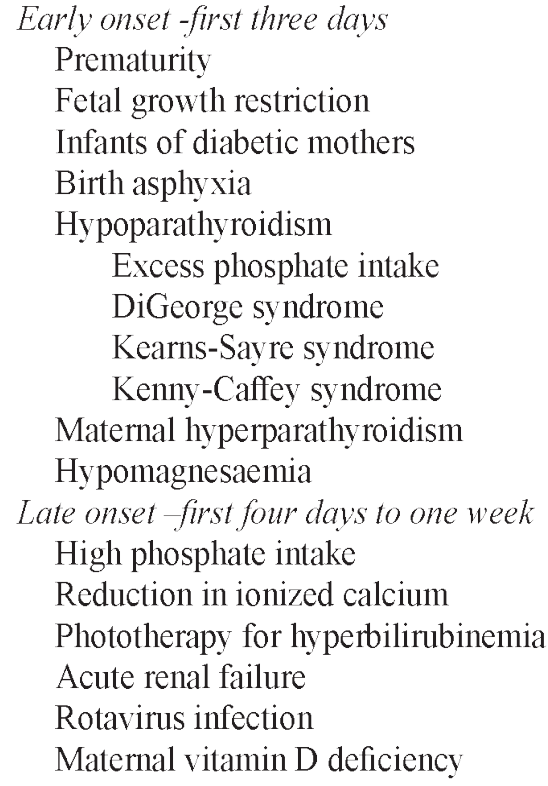

\section{CASE REPORT}

A term male neonate weighing $3.3 \mathrm{~kg}$ was born by normal vaginal delivery at a district hospital to a 29-year-old primigravida with no significant antenatal history. APGAR scores were 6 and 8 at 1 minute and 
5 minutes, respectively. He started crying vigorously after brief positive pressure ventilation and was ultimately able to shift with his mother to routine care. The infant exhibited difficulty breastfeeding due to ankyloglossia, thus he was discharged from the hospital with recommendations for expressed breast milk and formula supplementation. The infant was not given dairy milk, medications, or any herbal supplements.

On Day of Life (DOL) 10, infant presented to the Emergency Department with four episodes of focal seizures involving the right upper and lower extremity, decreased feeding, decreased urine output, and fever for one day. On initial examination, he was febrile with otherwise normal vital signs, neurological examination and blood glucose level.

The infant had another episode of focal seizure while under observation and was treated with phenobarbital and empirical antibiotics. Laboratory evaluation revealed calcium $4.6 \mathrm{mg} / \mathrm{dL}$ (Normal reference value is $8.6-10.8 \mathrm{mg} / \mathrm{dL}$ ), magnesium $1.2 \mathrm{mg} / \mathrm{dL}$ (Normal reference value is $1.8-2.6 \mathrm{mg} / \mathrm{dL}$ ), phosphorus $9.4 \mathrm{mg} / \mathrm{dL}$ (Normal reference value is $2.7-5.1 \mathrm{mg} / \mathrm{dL}$ ), sodium $146 \mathrm{mg} / \mathrm{dL}$, urea $166 \mathrm{mg} / \mathrm{dL}$, creatinine $2.4 \mathrm{mg} / \mathrm{dL}$. There was no evidence of sepsis and liver function tests were normal. $\mathrm{CT}$ of the brain was normal and CSF studies revealed no evidence of infection. Calcium was corrected with intravenous (IV) calcium gluconate injection $80 \mathrm{mg} / \mathrm{kg} /$ day for 48 hours, followed by $40 \mathrm{mg} / \mathrm{kg} /$ day for 24 hours $^{3}$.

By DOL 12, he was clinically stabilized with normal kidney function, no further seizure activity and normal serum electrolytes, except for calcium of 5.6 $\mathrm{mg} / \mathrm{dL}$ and magnesium of $1.3 \mathrm{mg} / \mathrm{dL}$. Phenobarbital was discontinued and IV calcium gluconate was restarted. Magnesium was also corrected by $50 \%$ magnesium sulfate $0.2 \mathrm{ml} / \mathrm{kg} /$ dose 12 hourly intramuscular injection for one day followed by $0.2 \mathrm{ml} / \mathrm{kg} /$ day intravenous infusion for two days. Further work up for hypocalcemia revealed hypovitaminosis D with 25 Hydroxy Vitamin D of $39.4 \mathrm{nmol} / \mathrm{L}$ (normal reference value is $75-250 \mathrm{nmol} / \mathrm{L}$ ). The ultrasonography of the thyroid gland was normal, but parathyroid glands were not visualized. Parathyroid Hormone (PTH) level was found to be $71.5 \mathrm{pg} / \mathrm{mL}$ (normal reference value is
$14.00-72.00 \mathrm{pg} / \mathrm{mL})$.

Serum calcium and magnesium normalized by DOL 22. MRI of brain done at DOL 23 showed mild meningeal enhancement but otherwise was normal. Albumin level was $3.4 \mathrm{mg} / \mathrm{dL}$ on DOL 25 and albumin supplement has not been given at any point of time.

He was started on vitamin D supplements and discharged from the hospital at DOL 31. Although the recommended dose is 2,000 IU daily for 6-12 weeks ${ }^{5}$, he was discharged on $1,600 \mathrm{IU}$ of Vitamin D in two formulations: a calcium supplement and a multivitamin drop. There is currently no separate Vitamin D syrup formulation available in Bhutan.

His mother's blood test revealed hypovitaminosis D (25-hydroxyVitamin D of 26.9 $\mathrm{nmol} / \mathrm{L}$ ) and hypocalcemia of $4.70 \mathrm{mg} / \mathrm{dL}$. She received calcium and vitamin B complex, iron and folic acid supplements during her pregnancy but did not receive any vitamin D supplements. She previously used to apply sunscreen whenever she went outside, but she stopped using sunscreen after she conceived. She is currently advised to take cholecalciferol granules 60,000 units weekly and shelcal (calcium carbonate $1250 \mathrm{mg}$ and vitamin D3 250IU) 500mg daily.

\section{DISCUSSION}

The differential diagnosis for neonatal seizures includes many entities (Table 1), but this case highlights the importance of rapid evaluation for reversible causes of neonatal seizures including hypoglycemia and hypocalcemia. Additionally, this infant presented with dehydration due to feeding problems, fever, and acute kidney injury. He continued to have focal seizure despite treatment with antiepileptics. Later, laboratory reports showed that this patient had late-onset hypocalcemia as onset of symptom was beyond one week of life (Table 2). A thorough medical evaluation allowed us to rapidly eliminate possible etiologies and arrive at a diagnosis of hyperphosphatemic hypocalcemia due to vitamin $\mathrm{D}$ deficiency. Vitamin $\mathrm{D}$, parathyroid hormone, and calcitonin are the primary drivers of calcium homeostasis. However, ionized calcium levels can be acutely altered by acidosis, which increases the level; or by phosphorus, bicarbonate, citrate, or alkalosis, all 
of which decrease ionized calcium. When considering the differential diagnosis for hypocalcemic seizures, all of these factors affecting calcium may be involved. Vitamin D has a large influence on the intestinal absorption of calcium. PTH increases within minutes in response to hypocalcemia and increases reabsorption of calcium in the kidneys and bone. Calcitonin acts to decrease bone reabsorption when calcium levels are normal ${ }^{4}$. In the care of this patient, the interactions between calcium, phosphorus, and magnesium, as well as the influence of vitamin D and PTH, were all evaluated.

Hyperphosphatemia contributes to hypocalcemia by binding calcium and acutely decreasing ionized calcium levels, in addition to decreasing intestinal calcium absorption. The most common etiology of hyperphosphatemia in neonates is an exogenous phosphate load from the ingestion of formula or cow's milk, which have a higher phosphate content than breastmilk (e.g. Enfamil $287 \mathrm{mg} / \mathrm{L}$, Lactogen 206mg/L, Breastmilk 143mg/L)4,6. As stated above, there was no history of cow's milk intake in this infant, although he was taking formula due to breastfeeding problems related to ankyloglossia. Given that he had low total calcium and the hypocalcemia did not improve after phosphorus levels decreased, it is unlikely that hyperphosphatemia alone was the primary cause.

Hypomagnesemia and hypocalcemia frequently occur together ${ }^{4}$. Magnesium can affect calcium levels, as it influences release of parathyroid hormone and tissue sensitivity to $\mathrm{PTH}^{7}$. It is unlikely that this was the primary cause for hypocalcemia in this patient, due to the fact that he continued to have hypocalcemia despite treatment with magnesium sulfate (Figure 1). Typically, patients with hypocalcemia related to primary hypomagnesemia will have an increase in PTH and calcium levels after treatment of the hypomagnesemia ${ }^{7}$.

The patient's hypocalcemia persisted even after correction of his pre-renal acute kidney injury and electrolyte abnormalities. Therefore, PTH

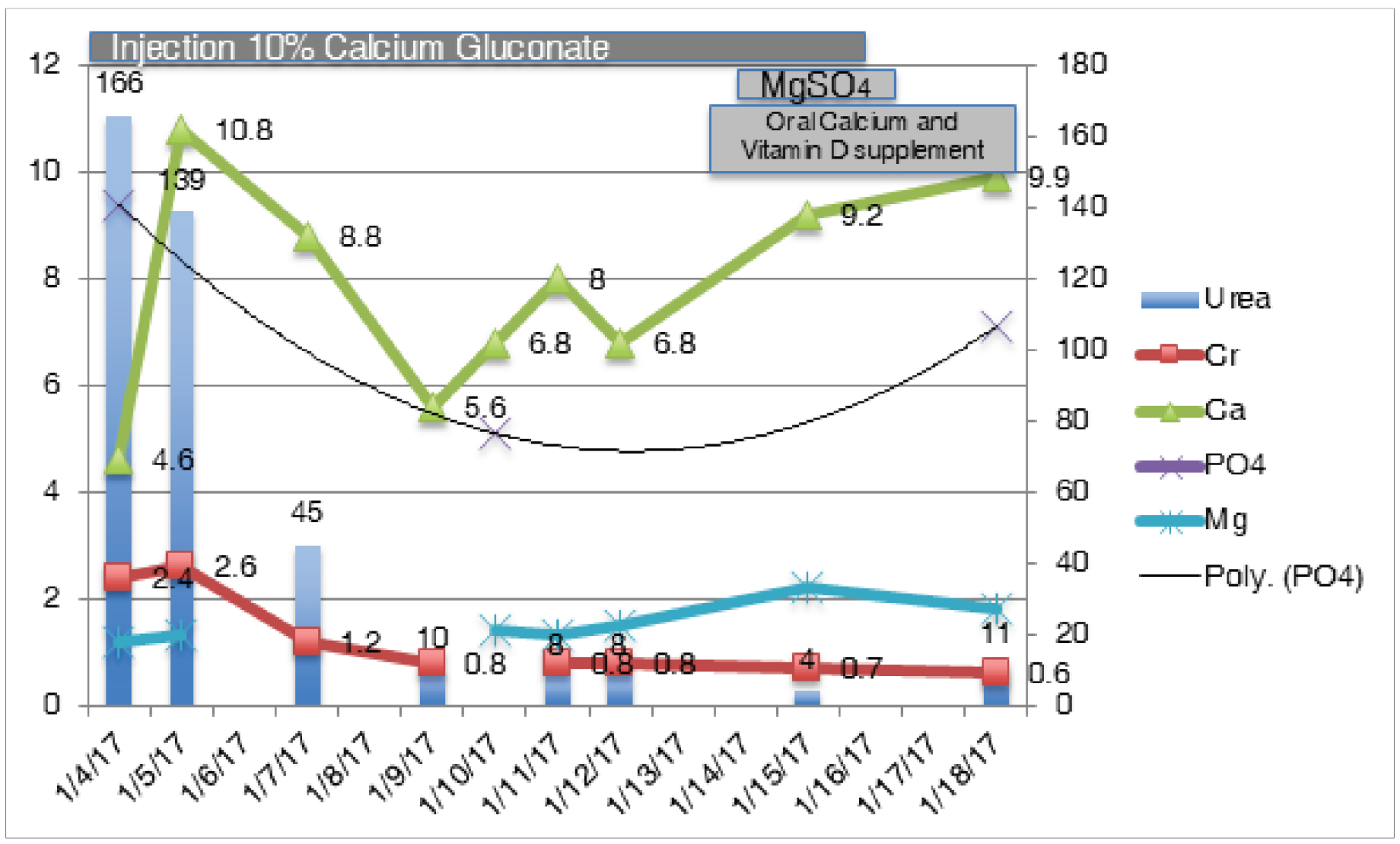

Figure 1. Clinical course 
and Vitamin D levels were tested, which revealed hypovitaminosis D in the mother and child. Maternal vitamin $\mathrm{D}$ status is the primary determinant of vitamin D levels antenatally and in the newborn period ${ }^{1,4,8}$. While adults with hypovitaminosis D almost always present with hypophosphatemia, it has been reported that neonates with hypovitaminosis D may present with low, normal, or high phosphate levels 9 . The literature suggests that neonates can present with low, normal, or high parathyroid hormone possibly due to the immaturity of the calcium- vitamin D- PTH axis ${ }^{9}$. In addition, they can present with severe hypocalcemia, bone demineralization, or rickets and are at risk for poor growth ${ }^{8}$.

To our knowledge, this is the first case report of hypovitaminosis $\mathrm{D}$ in a patient in Bhutan. The severity of this patient's initial presentation, with hypocalcemic seizures, highlights the importance of including hypovitaminosis $\mathrm{D}$ in a differential diagnosis of late onset of neonatal seizures. Maternal vitamin $\mathrm{D}$ deficiency is the most important risk factor for neonatal vitamin $\mathrm{D}$ deficiency ${ }^{4}$, which has been associated with small for gestational age newborns, as well as low muscle and bone mass that may persist into childhood $^{11,12}$. Hypovitaminosis D has been associated with an increased risk of cardiovascular disease ${ }^{10}$ and in pregnant women, an increased risk of gestational diabetes, preeclampsia and bacterial vaginosis ${ }^{11}$.

There are no data available on vitamin D levels in Bhutan; however, vitamin D deficiency is prevalent in our neighboring countries: Thailand has $41.5 \%$ insufficiency and $34 \%$ deficiency, with variations depending on latitude and season ${ }^{13}$; Bangladesh has 12$83 \%$ deficiency; India has $27-42 \%$ deficiency ${ }^{14}$. Based on data from South Asia, it is likely that the Bhutanese population could be at risk as well, given a national dress that covers most skin, darker skin tone, and high rates of exclusive breastfeeding in a population increasingly working indoors - all factors known to increase the risk of hypovitaminosis $\mathrm{D}^{14}$. This case highlights the need for prevalence studies in Bhutan to strengthen the recommendations of testing and supplementation for pregnant women and neonates. It also highlights the need for a liquid formulation of Vitamin D supplementation in pharmacies or on the essential drug list for the country.

\section{ACKNOWLEDGEMENTS}

We would like to acknowledge the patient's parents for their consent.

\section{REFERENCES}

1. Panayiotopoulos CP. Chapter 5, Neonatal Seizures and Neonatal Syndromes. In: The Epilepsies: Seizures, Syndromes and Management. Oxfordshire (UK): Bladon Medical Publishing; 2005. [Full Text]

2. Abrams SA. Neonatal hypocalcemia [Internet]. UpToDate; 2016 [cited 2017Jan]. [Full Text]

3. Ramesh Agarwal. 15 Hypocalcemia. In: AIIMS Protocols in Neonatology. $1^{\text {st }}$ ed. India: CBS Publishers \& Distributors; 2015. 191-201.

4. Avroy AF, Richard JM, Chapter 96: Disorders of Calcium, Phosphorus, and Magnesium Metabolism in the Neonate. In: Fanaroff and Martin's Neonatal-Perinatal Medicine: Diseases of the Fetus and Infant.10 ${ }^{\text {th }}$ ed. Abrams SA, Tiosano D. Philadelphia: Elsevier Sanders; 2015.

5. Misra M. Vitamin D insufficiency and deficiency in children and adolescents. UpToDate; 2016. [cited 2017 Feb]. [Full Text]

6. Koldobskiy M, Thompson J. Chapter 21: Nutrition and Growth. In: The HarrietLane Handbook. 20 $0^{\text {th }}$ ed. Philadelphia:Elsevier Saunders;2015. 485-522.

7. Koo, Winston WK, Reginald T. Chapter 36: Calcium and Magnesium Homeostasis. In: Avery's Neonatology: Pathophysiology \& management of the newborn, $6^{\text {th }}$ ed. Netherlands: Wolters Kluwer. 2005.

8. Wagner CL, Greer FR. Prevention of rickets and vitamin $\mathrm{D}$ deficiency in infants, children, and adolescents. Pediatrics. 2008;122 (5):1142-52. [PubMed | Full Text | DOI] 
9. Teaema FH, Ansari KA. Nineteen cases of symptomatic neonatal hypocalcemia secondary to vitamin D deficiency: A 2-year study. J Trop Pediatr. 2009;56(2):108-10. [PubMed | Full Text | DOI]

10. Wallis DE, Penckofer S, Sizemore GW. The "Sunshine Deficit" and Cardiovascular Disease. Circulation. 2008;118(14):1476-85. [PubMed | Full Text | DOI]

11. Chakhtoura M, Nassar A, Arabi A, Cooper C, Harvey N, Mahfoud Z, et al. Effect of vitamin D replacement on maternal and neonatal outcomes: a randomised controlled trial in pregnant women with hypovitaminosis D. A protocol. BMJ Open [Internet]. 2016;6(3):e010818. [Full Text | DOI]
12. Javaid MK, Crozier SR, Harvey NC, Gale CR, Dennison EM, Boucher BJ, et al. Maternal vitamin D status during pregnancy and childhood bone mass at age 9 years: a longitudinal study. The Lancet. 2006;367(9504):36- 43. [Full Text | DOI]

13. Pratumvinit $B$, Wongkrajang $P$, Wataganara $T$, Hanyongyuth S, Nimmannit A, Chatsiricharoenkul $\mathrm{S}$, et al. Maternal vitamin d status and its related factors in pregnant women in Bangkok, Thailand. PLoS One. 2015 Jun;10(7). [Full Text | DOI]

14. Arabi A, Rassi RE, Fuleihan GE-H. Hypovitaminosis D in developing countriesprevalence, risk factors and outcomes. Nat Rev Endocrinol. 2010 Oct6;6(10):550-61. [PubMed | Full Text | DOI] 